\title{
Repetitive acute cholangitis and pancreatitis due to Lemmel syndrome: A case report
}

\author{
Kazuki Takakura ${ }^{1}$, Shigeo Koido ${ }^{1}$, Mikio Kajihara ${ }^{1}$, Ryosuke Fukue ${ }^{1}$, Shinichiro Takami ${ }^{1}$, \\ Zensho I to ${ }^{1}$, Hiroko Kobayashi ${ }^{1}$, Yoshihiro Matsumoto ${ }^{1}$, Shintaro Tsukinaga ${ }^{1}$, Shunichi \\ Odahara ${ }^{1}$, Toyokazu Yukawa ${ }^{1}$, Kan Uchiyama ${ }^{1}$, Takeyuki Misawa ${ }^{2}$, Toshifumi Ohkusa ${ }^{1}$, Hisao \\ Tajiri ${ }^{1,3}$
}

1. Division of Gastroenterology and Hepatology, Department of Internal Medicine, The Jikei University School of Medicine, Tokyo, Japan. 2. Department of Surgery, The Jikei University School of Medicine, Tokyo, Japan. 3. Department of Endoscopy, The Jikei University School of Medicine, Tokyo, Japan.

Correspondence: Kazuki Takakura. Address: The Jikei University School of Medicine, 3-25-8 Nishi-shimbashi, Minato-ku, Tokyo 105-8461, Japan. Email: ktakakura@jikei.ac.jp

Received: March 3, 2014

DOI : $10.5430 /$ ijdi.v1n2p88
Accepted: April 25, $2014 \quad$ Online Published: May 15, 2014

URL: http://dx.doi.org/10.5430/ijdi.v1n2p88

\section{Abstract}

Duodenal diverticulum is a disease that clinicians commonly encounter in ordinary clinical work. Most cases are rarely regarded as clinical problems because of their asymptomatic progress. Among these cases, since Lemmel reported the clinical significance of juxtapapillary duodenal diverticula in 1934, describing them as Papillensyndrom, the effect of juxtapapillary duodenal diverticula on biliary-pancreatic diseases has been discussed due to the anatomical relationship of diverticula and the papilla of Vater. Although Lemmel syndrome's frequency, impact on each disease and operative procedure have been reported in Japan, its clinical significance is still unclear. Until now, no guidelines regarding the therapeutic indication of juxtapapillary duodenal diverticula have been established, so a suitable therapeutic strategy needs to be selected for each patient.

Here, we report a case of 60-year-old Japanese woman who repeatedly developed acute cholangitis and pancreatitis in a short period due to Lemmel syndrome, which was comprehensively diagnosed based on various imaging findings.

\section{Keywords}

Lemmel syndrome, Juxtapapillary duodenal diverticula, Obstructive jaundice, Pancreatitis

\section{I ntroduction}

Duodenal diverticulum has been reported in large numbers around the world since Chomel et al. reported in $1710^{[1]}$. Duodenal diverticulum is a disease that clinicians commonly encounter in ordinary clinical work. Postmortem studies have found that the prevalence of duodenal diverticulum ranges from $11 \%$ to $22 \%{ }^{[2]}$. There are few serious complications, such as diverticulitis, bleeding and perforation, and most cases are rarely regarded as clinical problems because of their asymptomatic progress. However, since Lemmel reported the clinical significance of juxtapapillary duodenal diverticula (JPDD) in 1934, describing them as Papillensyndrom ${ }^{[3]}$, the effect of JPDD on biliary-pancreatic diseases has been discussed due to the anatomical relationship of diverticula and the papilla of Vater. The majority of duodenal diverticula are located within $2 \mathrm{~cm}$ of the papilla of Vater and are called JPDD ${ }^{[4]}$. 
Although Lemmel syndrome's frequency, impact on each disease and operative procedure have been reported in Japan, its clinical significance is still unclear.

Here, we report certain additional considerations following our observation of a patient who repeatedly developed acute cholangitis and pancreatitis in a short period due to Lemmel syndrome, which was comprehensively diagnosed based on various imaging findings.

\section{Case presentation}

A 60-year-old Japanese woman was admitted to our hospital due to epigastralgia and pyrexia of 38 degrees Celsius. Her past medical history included paroxysmal atrial fibrillation, hyperlipidemia and sleep apnea syndrome.

In her physical examinations, the bulbar conjunctiva was icteric, and her body mass index exceeded 30, which were remarkable abnormal findings. The hepatobiliary tract enzyme levels on admission were elevated.

The laboratory data were as follows: total bilirubin, $3.7 \mathrm{mg} / \mathrm{dL}$; direct bilirubin, $2.4 \mathrm{mg} / \mathrm{dL}$; AST, $411 \mathrm{IU} / \mathrm{L}$; ALT, 421 IU/L; $\gamma$-GTP, 477 IU/L; S-AMY, 826 IU/L; lipase, 1041 IU/L; WBC, 13500/ $\mu \mathrm{L}$; and CRP, $6.8 \mathrm{mg} / \mathrm{dL}$.
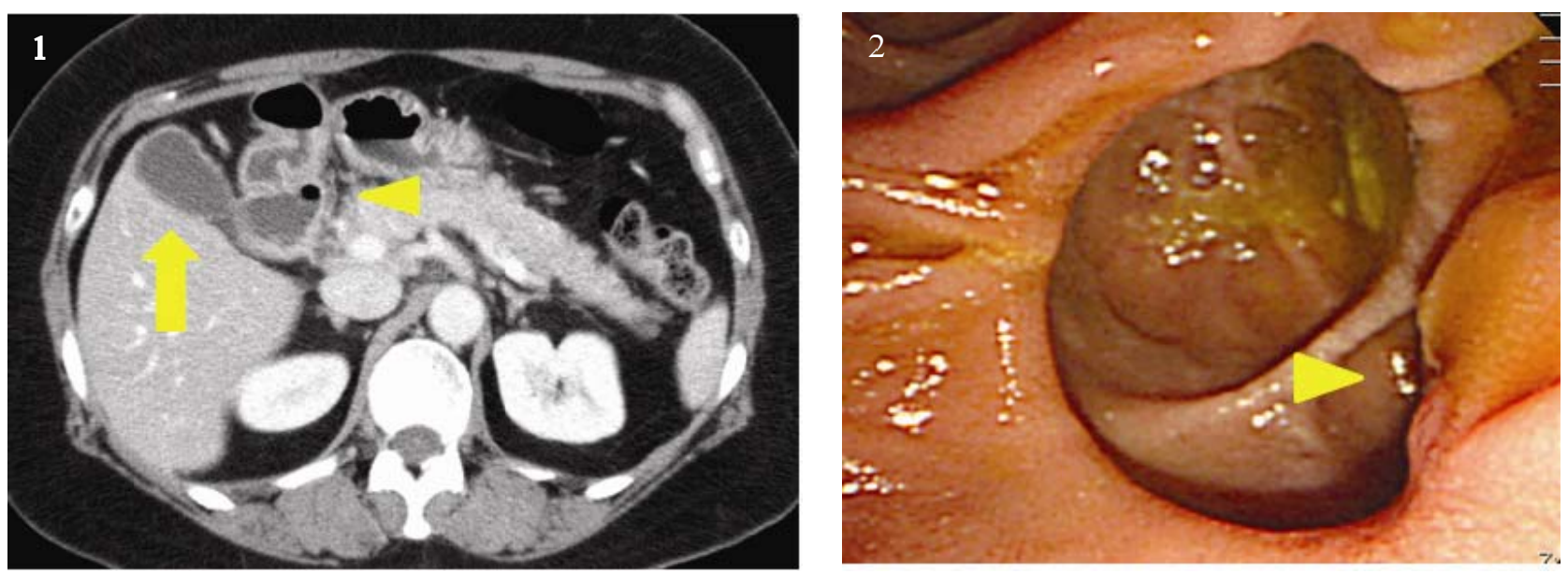

Figure 1. Abdominal dynamic computed tomography. Arrow: Gallbladder. Arrowhead: juxtapapillary duodenal diverticula.

Figure 2. Endoscopic finding: juxtapapillary duodenal diverticula. Arrowhead: the papilla of Vater by the side of a large diverticulum.

Figure 3. Magnetic resonance cholangiopancreatography.

No specific findings in the bile duct or pancreatic duct.

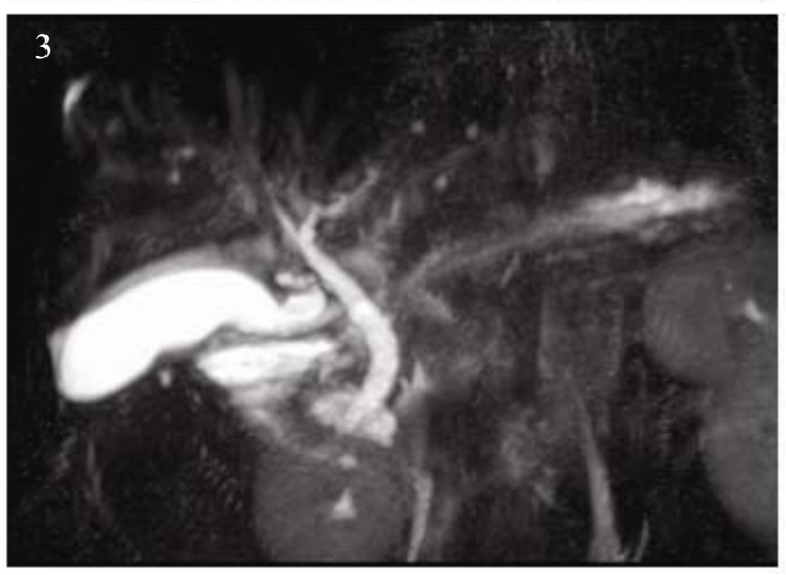

Abdominal dynamic computed tomography was performed on the day of admission and revealed JPDD, without evidence of an intra/extrahepatic bile duct stone or dilatation (see Figure 1). Subsequent esophagogastroduodenoscopy revealed a large diverticulum slightly on the oral side of the papilla of Vater in the descending portion of the duodenum (see Figure 2). An ultrasonographic study and endoscopic ultrasonography showed no notable abnormal findings in the related area. Magnetic resonance cholangiopancreatography revealed no defect suggesting a bile duct stone and no sign of pancreatic 
divisum (see Figure 3). Endoscopic retrograde cholangiopancreatography was not performed on the patient, considering the high technical risk.

Therefore, we could exclude other diseases, including bile duct stones, and comprehensively diagnosed the patient with Lemmel syndrome based on various imaging findings. Hence, we treated her conservatively, and she steadily improved. After 3 months, she was readmitted for the same complaint. However, no vigorous therapy was required for her recovery. She is currently being followed up in our outpatient department, and her progress after leaving the hospital is, fortunately, going well.

\section{Discussion}

By definition, Lemmel syndrome generally occurs as obstructive jaundice or acute pancreatitis, includes JPDD and does not include bile duct stones. In a broad sense, the syndrome can include a relationship between JPDD and the common bile duct in the form of cholestasis or retrograde bile duct infection and cases emerging from diverticula in the horizontal portion of the duodenum. The diagnosis of Lemmel syndrome is mainly performed by exclusion diagnosis at present, and it is important to exclude bile duct stones.

According to a previous report from Japan, the rate of complication for gallstones is approximately $30 \%$ and for the common bile duct is approximately $45 \%$ among cases having JPDD. Additionally, the rate of complication for acute pancreatitis is reported approximately $12 \%$ and for chronic pancreatitis is approximately $5 \%$. Landor et al. indicated the significance of JPDD and reported that the rate of complication for gallstones in the descending part of the duodenum is $31.1 \%$ and in the distal part is $13.4 \%{ }^{[5]}$. The mechanism of pathogenesis is conceivably due to naturally occurring pressure on the the common bile duct and pancreatic duct due to 1) intraduodenal pressure increases; 2) food debris flowing into a diverticulum; and 3) food debris, bile and pancreatic juice inside the diverticulum stimulating the papilla chronically and ultimately leading to papillary muscle dysfunction. Mcsherry et al. also mentioned that it is easy to develop lithogenesis and retrograde infection of the bile duct in the case of the common bile duct flowing directly into a diverticulum ${ }^{[6]}$. In the present case, acute cholangitis and pancreatitis occurred in a short period, even though there were JPDD, but not both a common bile duct stone and a gallbladder stone, in various imaging findings. Therefore, we comprehensively diagnosed the patient with Lemmel syndrome in reference to certain reported cases ${ }^{[7,8]}$.

The therapeutic strategies for Lemmel syndrome include surgical resection, endoscopic intervention and conservative medical treatment. Lemmel syndrome is currently considered as a disease that must be treated by diverticulectomy. However, cases that are difficult to resect because of contact with a diverticulum and the common bile duct or pancreatic duct are operated by diverticuloplasty or diverticular inversion. Moreover, in the case of certain conditions, such as diverticulosis or a diverticulum buried in the pancreatic head, a Billroth II operation is performed. If the patient selects a less invasive treatment or if surgical resection is difficult, we perform an endoscopic intervention, such as endoscopic sphincterotomy or endoscopic papillary balloon dilatation, or treat conservatively. In the present case, it was difficult to cannulate the common bile duct because of the anatomical relationship of the diverticulum and the papilla of Vater. Thus, we selected a conservative medical treatment.

Until now, guidelines regarding the therapeutic indication of JPDD have not been established, so we must select a suitable therapeutic strategy for each patient. A surgical operation is still the main choice ${ }^{[9,10]}$, but we should consider the patient's quality of life and requests because Lemmel syndrome is a benign disease. In the case of choosing conservative treatment, it is necessary to ensure that the patient does not experience recurrences or the subsequent occurrence of stenosis in the bile duct, leading to obstructive jaundice, or cholangiocarcinoma. 
In conclusion, we observed a patient with Lemmel syndrome who repeatedly developed acute cholangitis and pancreatitis in a short period. Fortunately, the patient recovered smoothly, and her progress after leaving the hospital is going well, without vigorous therapy.

\section{References}

[1] Chomel JBL. Historie de L'Academie Royal, Institute de France, Academie des science. Paris: 1710. 3p.

[2] Ackerman W. Diverticula and variations of the duodenum. Ann Surg 1943; 117: 403-13. http://dx.doi.org/10.1097/00000658-194303000-00007

[3] Lemmel G. Die klinische Bedeutung der Duodenal Divertikel. Arch f Verd-krkht 1934; 46: 59-70. http://dx.doi.org/10.1159/000196978

[4] Egged A, Teichmann W, Wittmann DH. The pathologic implication of duodenal diverticula. Surg Gynecol Obstet 1982; 154: 62-4.

[5] Landor JH, Fulkerson CC. Duodenal diverticula relationship to biliary tract disease. Arch Surg 1966; 96: 182-8. http://dx.doi.org/10.1001/archsurg.1966.01330010184023

[6] Mcsherry CK,Glenn F. Biliary tract obstruction and duodenal diverticula. Surg Gynecol Obstet 1970; 130(5): 829-36.

[7] Ono M, Kamisawa T, Tu Y, et al. MRCP and ERCP in Lemmel Syndrome. JOP. J Pancreas 2005; 6(3): 277-8.

[8] Doai K, Uchiyama K, Kuniyasu Y, et al. MR cholangiopancreatography of Mirizzi syndrome and Lemmel syndrome. Nihon Rinsho 1998; 56(11): 2933-8. (Japanese)

[9] Manny J, Muga M, Eyal Z. The continuing clinical enigma of duodenal diverticulum. Am J Surg 1981; 142: 596-600. http://dx.doi.org/10.1016/0002-9610(81)90434-7

[10] Pinotti HW, Tacla M, Pontes JF, et al. Juxta-Ampullar duodenal diverticula as cause of bilio-pancreatic disease. Digestion 1971; 4: 353-61. http://dx.doi.org/10.1159/000197140 